\title{
Effectiveness of highly purified urofollitropin treatment in patients with idiopathic azoospermia before testicular sperm extraction
} \author{
Sergio Serni ${ }^{1}$, Marco Carini ${ }^{1}$, Alessandro Natali ${ }^{1}$, Mauro Gacci ${ }^{1}$ \\ ${ }^{1}$ Department of Urology, AOU Careggi, University of Florence, Florence - Italy \\ ${ }^{2}$ Department of Urology, University of Catania, Catania - Italy \\ ${ }^{3}$ Department of Urology, University of Turin, Turin - Italy \\ ${ }^{4}$ Department of Urology, University of Naples Federico II, Naples - Italy \\ ${ }^{5}$ Department of Urology, University of Pisa, Pisa - Italy
}

Andrea Cocci ${ }^{1}$, Gianmartin Cito ${ }^{1}$, Giorgio Ivan Russo ${ }^{2}$, Marco Falcone ${ }^{3}$, Marco Capece ${ }^{4}$, Massimiliano Timpano ${ }^{3}$, Pier A. Della Camera ${ }^{1}$, Simone Morselli ${ }^{1}$, Giovanni Tasso ${ }^{1}$, Girolamo Morelli ${ }^{5}$, Giuseppe Morgia ${ }^{2}$, Andrea Minervini ${ }^{1}$,

\begin{abstract}
Introduction: Recent evidences demonstrated that male factor alone is responsible for about $30 \%$ cases of infertility. Human follicle-stimulating hormone (hFSH) has been introduced to increase sperm concentration, spermatogonial population, or both natural or assisted pregnancy rates (PRs) in oligozoospermic subjects with normal concentrations of gonadotropins.

Methods: Fifty infertile men affected by idiopathic azoospermia were enrolled in this study, after undergoing medical history, physical and clinical examination, baseline semen parameters and hormonal plasma concentrations. Inclusion criteria were infertility for at least 2 years, idiopathic azoospermia, $\mathrm{FSH}<12 \mathrm{mlU} / \mathrm{ml}$. Twenty-five patients were allocated to treatment with hFSH three times/week per 3 months (Fostimon), and 25 patients underwent just testicular sperm extraction (TESE) without medical treatment. All patients underwent, after 3 months, assisted reproduction techniques (ARTs) with TESE. The primary outcome was represented by the differences in the sperm retrieval rate (SRR) between groups, while the secondary outcomes were the differences in PR and fertilization rate (FR).

Results: We observed a PR of $15 \%(3 / 25)$ and $28 \%(7 / 25)$ in control and treated group, respectively. SRR after medical treatment and ART was $24 \%(6 / 25)$, while in the control group was $12.5 \%(2 / 25)$. The sperm in the ejaculate of five patients (20\%) after medical treatment exhibited a mean concentration of 0.9 million $/ \mathrm{ml}$ and a mean motility of $12 \%$. The FR was significantly greater in the treatment group with respect to the control group, $30 \%$ and $20 \%$, respectively.

Conclusions: FSH treatment showed greater efficacy rather than control by increasing the rate of PR and FR in azoospermic patients who underwent TESE.
\end{abstract}

Keywords: Assisted reproductive technologies, Azoospermia, Follicle-stimulating hormone, Hormone therapy

\section{Introduction}

Recent evidences have clearly demonstrated that male factor alone is responsible for nearly $30 \%$ cases of infertility (1).

Accepted: June 9, 2017

Published online:

Corresponding author:

Gianmartin Cito, MD

Department of Urology

AOU Careggi, University of Florence

Largo Brambilla, 3

50134 Florence, Italy

gianmartin.cito@gmail.com
Fortunately, the introduction of assisted reproduction techniques (ARTs), in particular the intrauterine insemination (IUI) and the in vitro fertilization (IVF), have widen the spectrum of the therapeutic options for infertile couples.

Human follicle-stimulating hormone (hFSH) has been introduced in order to increase sperm concentration, spermatogonial population, or both natural or assisted pregnancy rates in oligozoospermic subjects with normal plasma concentrations of gonadotropins (2).

On the basis of these considerations, some uncontrolled studies have shown an improvement of seminal parameters and/or an increase of pregnancy rates in infertile men with normogonadotropic oligozoospermia or azoospermia treated with different doses of FSH (3). 
[AU: As per the journal style, the short title should not be more than 40 characters including spaces. Please check and confirm the edited short title for correctness.]

Moreover, in order to minimize testicular damage during testicular sperm extraction (TESE), some researchers have sought to evaluate the role of hormone therapy for restoring spermatogenesis in men with particular idiopathic azoospermia (4). In fact, although the treated patients may remain azoospermic, the therapy may increase the retrieval rates of TESE.

However, considering the weakness of scientific evidences in this field of the reproductive medicine, we structured a prospective study aiming to evaluate the safety and efficacy of treatment with highly purified human-derived folliclestimulating hormone (HP Urofollitropin) in patients with idiopathic azoospermia before TESE.

\section{Methods}

A total of 50 men referred to our referral center for couple infertility and affected by idiopathic azoospermia were enrolled in this prospective study. All patients underwent detailed medical anamnesis and clinical examination before recruitment. The baseline semen parameters and hormonal levels (FSH, luteinizing hormone, total testosterone and inhibin $\mathrm{B}$ ) were collected for each patient. Inclusion criteria were as following: couple infertility for at least 2 years, idiopathic azoospermia, understood as a form of azoospermia with unknown etiology, $\mathrm{FSH}<12 \mathrm{mIU} / \mathrm{ml}$, and testicular volume between 12 and $20 \mathrm{ml}$. Exclusion criteria were drug hypersensivity, abnormal bleeding, adrenal dysfunction, intracranial lesion, and uncontrolled thyroid.

The results of two separate semen analyses confirmed the diagnosis of azoospermia (i.e., no spermatozoa after examination of the centrifuged pellet). The two sperm samples were analyzed according to the World Health Organization 2010 guidelines.

One group of 25 patients was allocated to the treatment with hFSH, injected subcutaneously three times/week per 3 months (Fostimon 150 IU/injection) diluted with physiological saline, while another group of 25 patients, retrospectively analyzed, was treated just with TESE without a previous medical treatment.

Finally, all patients underwent a TESE procedure in an attempt to retrieve testicular sperm suitable for intracytoplasmatic sperm injection (ICSI).

Sperm was researched by placing in a petri dish filled with $0.5 \mathrm{ml}$ of human tubal fluid medium, minced and shredded using sterile glass slides. Then, each sample was examined immediately by placing a small droplet.

The primary outcome was represented by the differences in the sperm retrieval rate between groups, while the secondary outcomes were the differences in terms of pregnancy rate and fertilization rate, respectively. Drug safety was evaluated by pre- and post-treatment blood biochemistry and hematology, and recording all side effects.

Continuous variables are presented as mean \pm standard deviation (SD) and differences between groups were tested by Student's independent $t$-test or Mann-Whitney $U$-test according to their distribution, respectively (normality of variables' distribution was tested by Kolmogorov-Smirnov test). Categorical variables were tested with the Chi-square analysis. All tests were completed using SPSS v. 19 software (SPSS
Inc, IBM Corp, Somers, New York, USA). For all statistical comparisons, significance was considered as $\mathrm{p}<0.05$.

\section{Results}

Table I lists the characteristics of patients at the enrollment. As clearly shown, groups were well balanced at the baseline. As summarized in Table II, the sperm in the ejaculate of five patients (20\%) after medical treatment exhibited a mean (SD) concentration of 0.9 (0.7) million/ml and a mean (SD) motility of $12 \%$ (10\%). We noted that the sperm retrieval rate after medical treatment and TESE was $24 \%$ (6/25), while in the control group was $12.5 \%(2 / 25)$. The fertilization rate was significantly greater in the treatment group with respect to the control group, $30 \%$ and $20 \%$, respectively $(p<0.05)$. At the final follow-up, we observed a pregnancy rate of $15 \%$ $(3 / 25)$ and $28 \%(7 / 25)$ in control and treated group, respectively $(p<0.05)$.

Finally, we did not find any statistically differences in age, testicular volume, initial serum total testosterone, or FSH in patients with successful sperm retrieval as compared with those without sperm found at TESE.

\section{Conclusion}

The aim of the present pilot study is to evaluate the effects of hFSH administration before TESE in men affected by infertility secondary to idiopathic azoospermia.

It has been previously published by Schill et al, who noted an increase in mean sperm concentration of 15.3 million/ml

TABLE I - Baseline characteristics of included patients

\begin{tabular}{lcc}
\hline & Control & Treated \\
\hline Number of subjects & 25 & 25 \\
Age of patients, years (mean \pm SD) & $34.0 \pm 4.0$ & $35.5 \pm 4.3$ \\
Infertility duration, years (mean \pm SD) & $3.2 \pm 2.2$ & $3.4 \pm 2.5$ \\
Body mass index, kg/m² (mean \pm SD) & $22.3 \pm 1.8$ & $22.5 \pm 2.0$ \\
Ejaculate volume, ml (mean \pm SD) & $3.2 \pm 2.4$ & $3.1 \pm 2.0$ \\
Testosterone, nmol/I (mean \pm SD) & $12.0 \pm 2.4$ & $12.8 \pm 3.5$ \\
LH, mIU/ml (mean \pm SD) & $5.3 \pm 2.8$ & $5.5 \pm 2.5$ \\
FSH, mIU/ml (mean \pm SD) & $7.7 \pm 2.2$ & $7.8 \pm 2.4$ \\
\hline
\end{tabular}

TABLE II - Primary and secondary outcomes after treatment

\begin{tabular}{lccc}
\hline & Control & Treated & p \\
\hline Sperm in semen, \% & $0(0)$ & $5(20)$ & $<0.05$ \\
Pregnancy rate, $\mathrm{n}(\%)$ & $3(15)$ & $7(28)$ & $<0.05$ \\
Sperm retrieval rate, $\mathrm{n}(\%)$ & $7(28)$ & $10(40)$ & $<0.05$ \\
Fertilization rate, $\mathrm{n}(\%)$ & $5(20)$ & $8(30)$ & $<0.05$ \\
\hline
\end{tabular}


after 3 months of treatment with human chorionic gonadotropin (hCG) and human menopausal gonadotropin (hMG) in men with idiopathic oligospermia and normal levels of gonadotropin (5).

In the past decade, andrologists had clomiphene citrate, hCG and hMG as treatment for idiopathic oligospermia, reporting improvement in semen quality and increased pregnancy rates among the partners of men in whom these agents were administered $(6,7)$.

A systematic review and meta-analysis showed a significant $4 \%$ increase in live-birth rate with the use of hMG, when compared with recombinant FSH ( $\mathrm{rFSH})$, following a long downregulation protocol in IVF-ICSI treatment cycles (8). Hussein et al showed that for the patients who did not respond to clomiphene citrate as sole therapy and were managed with addition of hCG with or without hMG, $60 \%$ of patients ultimately had sperm available for ICSI. Authors observed that these patients had an approximately doubled sperm retrieval rate compared with the control group, and the increased availability of sperm from each group of medically treated patients compared with the control group was significant $(p<0.05)$.

Herein, we demonstrate that patients treated with hFSH showed greater benefits than the control group in terms of pregnancy rate and sperm retrieval rate.

The physiologic regulation of spermatogenesis is still not completely understood. In particular, FSH is one of the major hormones produced by the anterior pituitary gland, activates the proliferation of the sertoli cells, and then induces the mitotic activity of the spermatogonia and supports cellular differentiation until the round spermatid stage (7). Recently, the use of hFSH or rFSH has been addressed with the aim to investigate the putative role of this hormone for the treatment of infertility. It is possible that germ cells increase in seminiferous tubule causing testicular volume to increase (8).

Ding et al studied a total of 354 men affected by idiopathic oligozoospermia from three medical centers: sperm number was significantly increased after treatment with FSH at doses of at least $200 \mathrm{IU}$, and the improvement was observed beginning at the third month. Moreover, $300 \mathrm{IU}$ recombinant human FSH (rhFSH) administration for 5 months improved the spontaneous pregnancy rate and ART pregnancy rate.

The clinical efficacy of FSH treatment could be secondary to the promotion of spermatogenesis in seminiferous tubules. The possible mechanisms that FSH maintains spermatogenesis may be (i) activating the proliferation of the sertoli cells; (ii) increasing the number of type A spermatogonia; (iii) inducing the mitotic activity of the spermatogonia and supporting cellular differentiation until the round spermatid stage $(9,10)$.

Some studies have suggested that rhFSH treatment could restore sperm structure and increased sperm DNA condensation in idiopathic infertile men (9).

However, our study has several limitations. First of all, the lack of placebo and the absence of an additional subanalysis (i.e., age adjusted, comorbidities adjusted) should be underlined. Moreover, there are no different types of FSH treat- ment, nor several doses and the short follow-up could have limited the evaluation of the pregnancy rate.

In conclusion, FSH treatment could be used to increase ART efficacy in infertile men with idiopathic azoospermia, but further studies, larger and placebo-controlled, are needed to confirm our preliminary data.

We demonstrated that FSH treatment showed greater efficacy rather than control by increasing the rate of pregnancy rate and fertilization rate in azoospermic patients who underwent TESE.

\section{Disclosures}

Financial support: The authors have not received any funding for this study.

Conflict of interest: All authors declare that there is no conflict of interest regarding the publication of this paper.

\section{References}

1. Macleod J, Gold RZ. The male factor in fertility and infertility. II. Spermatozoön counts in 1000 men of known fertility and in 1000 cases of infertile marriage. J Urol. 2017;197(2)(2S): S78-S91.

2. Garolla A, Ghezzi M, Cosci I, et al. FSH treatment in infertile males candidate to assisted reproduction improved sperm DNA fragmentation and pregnancy rate. Endocrine. 2017;56(2): 416-425.

3. Barbotin AL, Ballot C, Sigala J, et al. Pregnancy after intracytoplasmic sperm injection following extended sperm preparation and hormone therapy in an azoospermic man with maturation arrest and microlithiasis: a case report and literature review. Andrologia. 2017;49(5).

4. Schill WB, Jüngst $D$, Unterburger $P$, Braun $S$. Combined hMG/ hCG treatment in subfertile men with idiopathic normogonadotrophic oligozoospermia. Int J Androl. 1982;5(5):467-477.

5. Miyagawa Y, Tsujimura A, Matsumiya K, et al. Outcome of gonadotropin therapy for male hypogonadotropic hypogonadism at university affiliated male infertility centers: a 30-year retrospective study. J Urol. 2005;173(6):2072-2075.

6. Farrag A, Sagnella F, Pappalardo S, et al. The use of $r$-hFSH in treatment of idiopathic male factor infertility before ICSI. Eur Rev Med Pharmacol Sci. 2015;19(12):2162-2167.

7. Ding YM, Zhang XJ, Li JP, et al. Treatment of idiopathic oligozoospermia with recombinant human follicle-stimulating hormone: a prospective, randomized, double-blind, placebocontrolled clinical study in Chinese population. Clin Endocrinol (Oxf). 2015;83(6):866-871.

8. Coomarasamy A, Afnan M, Cheema D, van der Veen F, Bossuyt $\mathrm{PM}$, van Wely $\mathrm{M}$. Urinary $\mathrm{hMG}$ versus recombinant FSH for controlled ovarian hyperstimulation following an agonist long down-regulation protocol in IVF or ICSI treatment: a systematic review and meta-analysis. Hum Reprod. 2008;23(2):310-315.

9. Weinbauer GF, Behre HM, Fingscheidt U, Nieschlag E. Human follicle-stimulating hormone exerts a stimulatory effect on spermatogenesis, testicular size, and serum inhibin levels in the gonadotropin-releasing hormone antagonist-treated nonhuman primate (Macaca fascicularis). Endocrinology. 1991;129(4): 1831-1839.

10. de Kretser DM, Loveland KL, Meinhardt A, Simorangkir D, Wreford N. Spermatogenesis. Hum Reprod. 1998;13(Suppl 1):1-8. 